\title{
Paraquat poisoning and the lung pathology; vascular injury leading to thrombotic microangiopathy is the primary pathological event
}

\begin{abstract}
The authors present a case of a suicide death caused by ingestion of paraquat. The autopsy and histological examination highlighted pulmonary thrombotic microangiopathy and emphysematous change as the respiratory pathology that contributed to the cause of death. We show that thrombotic microangiopathy is the primary underlying pathology in paraquat poisoning.
\end{abstract}

Keywords: paraquat poisoning, thrombotic microangiopathy, alveolar capillaries, emphysematous changes, primary pathological event

\author{
Volume 5 Issue 6 - 2018 \\ Hubert Daisley, Arlene Rampersad, Oneka \\ Acco, Dawn Meyers \\ General Hospital,TrinidadWI
}

Correspondence: Hubert Daisley, General Hospital, San Fernando,Trinidad WI,Tel + I 8683990188 ,

Email prothdjr@aol.com

Received: October 24, 2018 | Published: November 16, 2018
Keywords: paraquat poisoning, thrombotic microangiopathy, alveolar capillaries, emphysematous changes, primary pathological event

\section{Introduction}

Paraquat is an agent that is used in suicide in many agricultural societies. ${ }^{1-3}$ Its ingestion is almost always fatal. Respiratory failure plays a significant role in the demise of these victims as pulmonary fibrosis ultimately occurs. The literature abounds with theories as to the pathogenesis of paraquat toxicity, most of which focuses on the inflammatory infiltrates and the pneumocytes' response in the lung in paraquat poisoning. As a result immunosuppressive and anti-fibrosis therapies have been tailored against these latter pathophysiological mechanisms, ${ }^{4,5}$ needless to say these therapies have been met with little success.

The vascular injury theories in paraquat poisoning have been subdued, ${ }^{6}$ but the findings in the case in discussion leaves no doubt that vascular injury with thrombotic microangiopathy is a major event in paraquat poisoning, and that therapy should be directed towards thrombotic microangiopathy.

\section{Case presentation}

A 27-year-old male presented to the A \& E department of the country hospital, some two hours after he had ingested approximately $400 \mathrm{mls}$ of paraquat, following a heated dispute with his female companion. He suffered with no chronic disease or psychiatric disorder.

While being administered oral activated charcoal in A\&E, he suffered a cardio-respiratory arrest from which he did not respond to active resuscitation. He was certified dead just 20 minutes after arrival to hospital. An autopsy was mandated since it was a coroner's case.

At autopsy, the body was that of a young male. There was cyanosis at the nail beds. His lips were stained green, and a pungent odour emanated from his nostrils and mouth. His lips were ulcerated. His stomach contained $400 \mathrm{mls}$ of paraquat and the mucosa was hyperemic.
The right and left lungs weighed 700 and $540 \mathrm{Gms}$ respectfully. They both were distended, congested and edematous. The heart, its coronaries, myocardium and valves were structurally unremarkable. The kidneys were edematous but were within the normal weight range, and so too were the adrenals, pancreas, and spleen. The brain weighed $1400 \mathrm{Gms}$ and was edematous. There were no macroscopic infarctions or hemorrhages. Tissue samples were taken from all organs for histological examination, and blood and stomach contents for toxicological examination.

The histological examination of the lungs using the H\&E stain revealed diffuse terminal arteriole and alveolar capillary thrombosis (Figure 1), emphysematous and atelectatic changes, destruction of alveolar walls, and rupture of sub pleural alveolar walls (Figure 2). There was very scant inflammation within alveolar walls. The heart, brain, and kidneys, showed multiple thrombi within the microvasculature.

\section{Discussion}

Paraquat, 1, 1'-dimethyl-4, 4'-bipyridylium dichloride, is a highly toxic and effective quaternary ammonium herbicide. It exerts its toxic effect through its redox cycle through the production of superoxide anions in organisms.?

It is very toxic to humans, and has been an agent of suicide in many agricultural communities. ${ }^{8}$ Death is often ensured from multiorgan failure, mainly respiratory failure occurring due to pulmonary fibrosis. Once ingested paraquat is absorbed and enters the blood stream and is distributed to all organs and tissues. The highest concentration of paraquat is seen in the lung due to the selective concentration gradient orchestrated by the polyamine transport system. ${ }^{9}$

Vascular endothelial cells, to protect and maintain their integrity and modulate blood flow, thrombosis and neural activity, produce nitric oxide. Diminishing the concentration of nitric oxide exposes the endothelial cell to injury. Endothelial injury, changes in the constituent of blood and stasis acting singularly or in combination, causes thrombosis according to Virchow's triad. ${ }^{10}$ 
Paraquat either by its direct toxic effect or by its production of superoxide radicles within the lung, or by the reaction of nitric oxide and superoxide radicles to produce peroxinitrite, causes oxidative endothelial cellular injury. ${ }^{11}$

The injury to endothelial cells so produced sets about a series of reactions, which causes thrombosis within terminal arterioles and alveolar capillaries.

VWF (Von Willebrand Factor), in addition to serving as a carrier of factor VIII, is the ligand for mediating platelet adhesion and aggregation at the sites of endothelial cells injury. VWF is synthesized in vascular endothelial cells and megakaryocytes and released in the plasma and sub endothelium in some vessels. In the circulation, the Von Willebrand polymer is converted to plasma multimers by a plasma metalloprotease namely ADAMTS13. This metalloprotease regulates VWF and platelet aggregation by its cleavage function. Its deficiency causes VWF released in endothelial cellular injury to bind avidly with platelets promoting thrombotic microangiopathy. ${ }^{12}$

The histological findings in the lung in the case in discussion show diffuse micro thrombi viz-thrombotic microangiopathy within terminal arterioles and alveoli capillaries (Figure 1). This thrombotic microangiopathy is the primary pathological event that occurs in the lung following ingestion of paraquat. There is resultant damage of alveolar wall with emphysematous and atelectatic changes. Damaged alveoli in sub pleural locations and disruption of the visceral pleura cause gases to leak into the pleural cavity leading to pneumothorax and in some cases pneumomediastinum (Figure 2). ${ }^{13,14}$

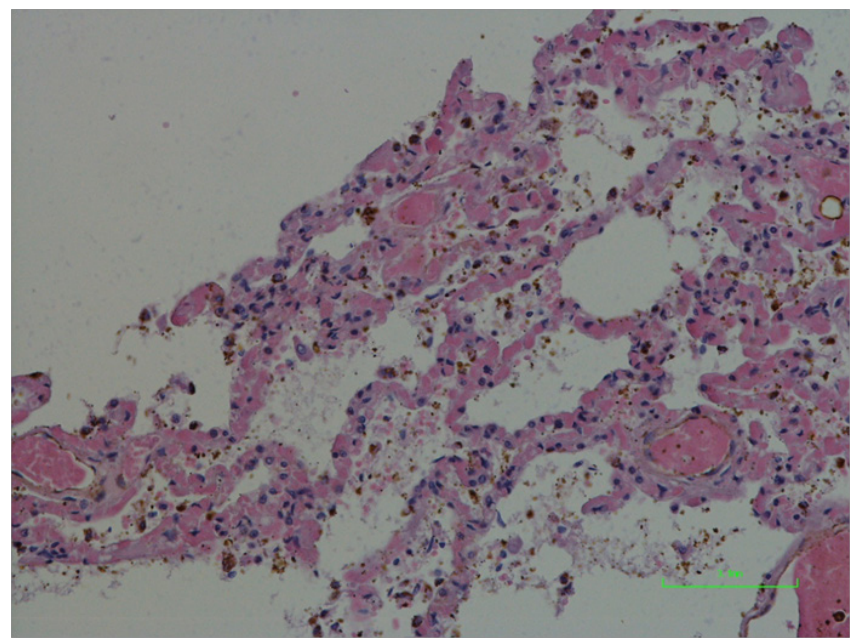

Figure I Show diffuse thrombosis of terminal arterioles and alveolar capillaries.

The inflammatory response, which leads to pulmonary fibrosis, is seen in those victims who survive for prolonged periods following paraquat ingestion. Pneumocytes proliferate in an effort to repair the damaged alveolar wall and produces collagen, which ultimately produces pulmonary fibrosis respiratory failure and death. ${ }^{15,16}$

These latter pathological processes have dominated the discussion in the medical literature with respect to the pulmonary pathology in paraquat poisoning, and overshadowed the relevance and prevalence of the vascular endothelial cellular injury role in paraquat toxicity. ${ }^{6}$

The pneumocytes' response and the inflammatory processes seen in the lung following paraquat poisoning are therefore secondary responses to the damage caused from the thrombotic microangiopathy in the terminal arterioles and alveolar capillaries.

Thrombotic microangiopathy is the primary event that occurs in paraquat toxicity. Thrombotic microangiopathy was also seen in the kidneys, heart and brain in the case in discussion, and is responsible for the multiple organ failure often experienced in paraquat toxicity. These latter events would be the subject of another discussion.

Therapy directed against thrombotic microangiopathy has been used successfully in treating patients with paraquat poisoning, ${ }^{17-19}$ which supports our finding that thrombotic microangiopathy is the major pathological event occurring in paraquat toxicity.

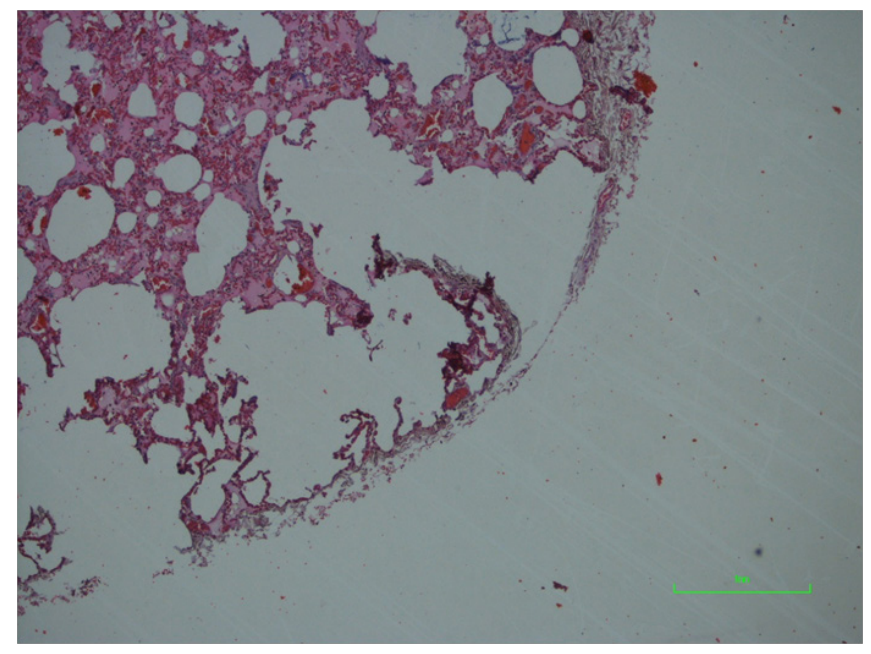

Figure 2 Shows destruction of alveolar wall and emphysematous changes in sub-pleural region.

\section{Conclusions}

Diffuse thrombosis of terminal arterioles and alveolar capillaries are the primary pathological event that occurs following acute paraquat toxicity. This leads to alveolar wall destruction, emphysematous changes and pneumothorax. Thrombotic microangiopathy is the primary underlying pathological event in all organs following paraquat toxicity.

\section{Author contributions}

The conceptualization was spear headed by Professor Hubert Daisley and the original draft preparation was done by same. The Autopsy examination of the case presented was done by Dr Arlene Rampersad and Dr Oneka Acco. Histologic examination and microphotography was performed by Dr Dawn Meyers. All above named authors contributed to the review and editing of the final manuscript.

\section{Funding}

This report received no funding.

\section{Acknowledgements}

None.

\section{Conflicts of interest}

The authors declare no conflict of interest. 


\section{References}

1. Hettiarachchi J, Kodithuwakku GCS. Pattern of Poisoning in Rural Sri Lanka. Int J Epid. 1989;18(2):418-422.

2. Hutchinson G, Daisley H, Simeon D, et al. High rates of paraquat-induced suicide in southern Trinidad. Suicide Life Threat Behav. 1999;29(2):186-191.

3. Cha ES, Chang SS, Gunnell D, et al. Impact of paraquat regulation on suicide in South Korea. Int J Epid. 2016;45(2):470-479.

4. Addo E, Poon-King T. Leucocyte suppression in treatment of 72 patients with paraquat poisoning. Lancet. 1986;1:1117-1120.

5. Wilks MF, Fernando R, Ariyananda PL, et al. Improvement in Surviva after Paraquat Ingestion Following Introduction of a New Formulation in Sri Lanka. PLoS Med. 2008;5(2):e49.

6. Dinis-Oliveira RJ, Duarte JA, Sanchez-Navarro A, et al. Paraquat poisonings: mechanisms of lung toxicity, clinical features, and treatment. Crit Rev Toxicol. 2008;38(1):13-71.

7. Blanco-Ayala T, Andérica-Romero AC, Pedraza-Chaverri J. New insights into antioxidant strategies against paraquat toxicity. Free Radic Res. 2014;48(6):623-640.

8. Gunnell D, Eddleston M, Phillips MR, et al. The global distribution of fatal pesticide self-poisoning: systematic review. BMC Public Health. 2007; 7:357.

9. Smith LL, Lewis CPL, Wyatt I, et al. The importance of epithelial uptake systems in lung toxicity. Environmental Health Perspectives. 1990;85:25-30
10. Kumar DR, Hanlin E, Glurich I, et al. Virchow's Contribution to the Understanding of Thrombosis and Cellular Biology. Clin Med Res. 2010;8(3-4):168-172.

11. Pacher P, Beckman JS, Liaudet L. Nitric oxide and peroxynitrite in health and disease. Physiol Rev. 2007;87(1):315-424.

12. Long Zheng X, Sadler JE. Pathogenesis of Thrombotic Microangiopathies. Annu Rev Pathol. 2008;3:249-277.

13. Daisley H, Barton EN. Spontaneous pneumothorax in acute paraquat toxicity. West Indian Med J. 1990;39(3):180-185.

14. Meyers D, Rampersad A, Christopher M, et al. Under diagnosed Paraquat Induced Pneumothorax and Pneumomediastinum, the "Daisley Barton Syndrome": A Clinical Feature Of Paraquat-Induced Acute Lung Injury. Int J Clin Pharmacol Toxicol. 2018;7(2):296-299.

15. Liu XL, Liu XR, Lu CZ. Acute lung injury mechanism and therapy induced by paraquat poisoning. J Acute Dis. 2017;6:260-263.

16. Sun S, Wang H, Zhao G, et al. Complement Inhibition Alleviates Paraquat-Induced Acute Lung Injury. Am J Respir Cell Mol Biol. 2011;45(4):834-842.

17. Wyrick-Glatzel J. Thrombotic thrombocytopenic purpura and ADAMTS-13: New insights into pathogenesis, diagnosis, and therapy. Lab Med. 2004;35(12):733-740.

18. Jang HN, Bae EJ, Hwang $\mathrm{K}$, et al. Hemolytic uremic syndrome associated with paraquat intoxication. J Clin Apher. 2014;29(3):183-186.

19. Rao R, Bhat R, Pathadka S, et al. Golden Hours in Severe Paraquat Poisoning-The Role of Early Haemoperfusion Therapy. Clin Diagn Res. 2017;11(2):OC06-OC08. 